\title{
Is the Abnormal Conduction Zone of the Left Atrium a Precursor to a Low Voltage Area in Patients with Atrial Fibrillation?
}

\author{
Hideyuki Kishima ${ }^{1}$, Takanao Mine ${ }^{1}$, Eiji Fukuhara ${ }^{1}$, Satoshi Takahashi², and Masaharu \\ Ishihara $^{1}$ \\ ${ }^{1}$ Hyogo Ika Daigaku \\ ${ }^{2}$ Kawasaki Hospital
}

July 29, 2020

\begin{abstract}
Background: The abnormal conduction zone (ACZ) in the left atrium (LA) has attracted attention as an arrhythmia source in atrial fibrillation (AF). We investigated the hypothesis that the ACZ is related to the low voltage area (LVA) or the LA anatomical contact areas (CoAs) with other organs. Methods and Results: We studied 100 patients (49 non-paroxysmal AF, 66 males, 67.9 \pm 9.9 years) who received catheter ablation for AF. High-density LA mapping during high right atrial pacing was constructed. Isochronal activation maps were created at 5-ms interval setting, and the ACZ was identified on the activation map by locating a site with isochronal crowding of [?]3 isochrones, which are calculated as [?] $27 \mathrm{~cm} / \mathrm{s}$. The LVA was defined as the following; mild $(<1.3 \mathrm{mV})$, moderate $(<1.0 \mathrm{mV})$, and severe LVA $(<0.5 \mathrm{mV})$. The CoAs (ascending aorta-anterior LA, descending aorta-posterior LA, and vertebrae-posterior LA) were assessed using computed tomography. The ACZ was linearly distributed, and observed in 95 patients (95\%). The ACZ was most frequently observed in the anterior wall region (77\%). A longer ACZ was significantly associated with a larger LA size and a prevalence of non-PAF. The 51.2+-36.2\% of ACZ overlapped with mild LVA, 32.9+-32.8\% of ACZ with moderate LVA, and 14.6+-22.0\% of ACZ with severe LVA. In contrast, only 25.6+$28.0 \%$ of ACZ matched with the CoAs. Conclusion: The abnormal conduction zone reflects LA electrical remodeling and may be a precursor finding of the low voltage zone and not the LA contact areas in patients with atrial fibrillation.
\end{abstract}

\section{Introduction}

Recurrent atrial fibrillation (AF) after catheter ablation (CA) is a common clinical problem. Main cause of AF recurrence is pulmonary vein (PV) reconnection. Nevertheless, some patients experience a recurrence even after complete PV isolation. These outcomes are likely explained by the development of an atrial arrhythmogenic substrate and a non-PV trigger. The previous study has failed to demonstrate the efficacy of CA with substrate modification for AF. ${ }^{1}$ Recent reports suggested that ablation for low voltage area (LVA) in addition to $\mathrm{PV}$ isolation improved procedural outcomes in patients with $\mathrm{AF} .^{2}$ However, these strategies for LVA have insufficient success rate.

It is known that atrial remodeling due to AF causes an increase in fibrosis, which is associated with a reduction in conduction velocity, and a consequent increase in susceptibility to $\mathrm{AF} .{ }^{3}$ Regions of slowed conduction have classically been considered markers of disease tissue that enable arrhythmia perpetuation, and that may sustain atrial arrhythmia. ${ }^{4}$ Previous studies reported that fibrosis of the left atrium (LA), resulting in conduction abnormality, appears to be associated with higher recurrence rates. ${ }^{5}$ Recently, Mouws et al reported that conduction abnormalities were associated with $\mathrm{AF} .{ }^{6}$ However, little has been reported on the abnormal conduction zone (ACZ) of the LA.

The Advisor HD grid mapping catheter (HD Grid, Abbott) was released in 2019. Using the HD Grid can create a detailed voltage map and local activation map. Furthermore, previous studies suggested that AF 
itself and other factors, such as compression from the external structures, can promote atrial remodeling. Hori et al investigated the relationship between the contact area $(\mathrm{CoA})$ of the LA with external anatomical structures in $22 \mathrm{AF}$ patients. They indicated that LVA had a strong association with CoA. ${ }^{7}$ Although the $\mathrm{ACZ}$ may be regions of interest in sustained $\mathrm{AF}$, detailed electrophysiological studies describing the nature of the ACZ are lacking. We focused on the ACZ, and investigated the characteristics of the ACZ and whether the ACZ was related to the LVA or the LA anatomical CoAs with other organs in patients with AF.

\section{Materials and Methods}

\section{Patients' population}

This study included patients with non-valvular AF who underwent high-density voltage mapping after PV isolation at the Hyogo College of Medicine and Kawasaki Hospital between August 2019 and April 2020. From this initial population, patients who had a structural heart disease, or patients requiring LA ablation other than PV isolation were excluded from the study. The remaining 100 patients were included in our study analyses. AF was categorized into two types; paroxysmal AF (PAF) and non-PAF. All antiarrhythmic drugs were withheld at least 5 half-lives before CA procedure.

All patients underwent transthoracic echocardiography (TTE) within 1-month preceding the procedure. LA diameter, $\mathrm{E}$ wave, deceleration time, LV diameter during end diastole, the prevalence of LV hypertrophy, $\mathrm{LV}$ ejection fraction, and E/e' ratio were assessed. LA diameter was indexed for body size by dividing by body surface area (BSA) calculated using the Dubois formula. LA size assessed by LA diameter/BSA was categorized into normal / mildly $\left(<2.6 \mathrm{~cm} / \mathrm{m}^{2}\right)$, moderately $\left(2.7-2.9 \mathrm{~cm} / \mathrm{m}^{2}\right)$, or severely $\left([?] 3.0 \mathrm{~cm} / \mathrm{m}^{2}\right)$ enlarged LA according to current recommendations. ${ }^{8}$ All patients were older than 18 years of age and provided written informed consent to the procedures. The study protocol complied with the Declaration of Helsinki and was approved by the appointed local ethics committee (The Ethics Review Board of Hyogo College of Medicine, Reference number: 3477).

\section{Catheter ablation procedure and mapping}

After 1 transseptal puncture, 2 long sheaths (Agilis and SL0; Abbott Medical) were introduced into both superior PV. A HD Grid were placed transseptally at the antrum of the target PVs. All patients underwent circumferential PV isolation guided. The circumferential ablation lines were created under the guidance of a 3-D mapping system (EnSite Precision system; Abbott Medical). After PV isolation, a detailed high-density map of LA was constructed by acquiring more than 2000 endocardial points in each patient during high right atrial pacing at a rate of $100 \mathrm{bpm}$ using $\mathrm{HD}$ Grid and the steerable sheath. Points acquired following ectopic beats and far-field ventricular electrograms were excluded from analysis. Automated 3-D mapping system (EnSite AutoMap module; Abbott Medical) was used to construct the LA geometry, voltage map, and isochronal activation map. The band pass filter was set at 30-500 Hz. To ensure the highest accuracy of the acquired atrial geometry, respiratory gating was performed, and the atrial geometry was acquired at high adjustment settings. In addition, high-density mapping was added at sites where LVA was recorded to exactly define the extent of the LVA. Endocardial contact was ensured by fluoroscopy, electrogram stability, and the 3-D mapping system. The highest amplitude bipolar signal among signals within a vicinity of less than $1 \mathrm{~mm}^{2}$ was reflected on the voltage map. Field scaling was applied to all maps. Every LA map was divided into the following six segments. The LVA was defined using cut-off values for bipolar peak-to-peak voltage as the following; mild $(<1.3 \mathrm{mV})^{9}$, moderate $(<1.0 \mathrm{mV})^{10}$, and severe LVA $(<0.5 \mathrm{mV})^{11}$. LVA size was manually measured on each cut-off value. The proportion of the mapped LA surface exhibiting low voltage was expressed as a percentage of the overall mapped surface area of LA within the isolation line, not including the ablation lesions in the surface area of the LA and anatomical structures such as the PV and mitral annulus. The mean bipolar voltage of global LA was calculated.

Isochronal activation maps were created using EnSite AutoMap module at 5-ms interval setting during high right atrial pacing at a rate of $100 \mathrm{bpm}$. An ACZ was identified on the activation map by locating a site with isochronal crowding of [?]3 isochrones in a 4-mm diameter tag, which are calculated as [?]27 cm/s. The cut-off value of ACZ was defined according to a previous report. ${ }^{12}$ Figure 1 shows a representative 
isochronal activation map and voltage maps. Global activation time for the LA was calculated by subtracting the earliest from the latest activation within the LA.

\section{Anatomical relationship between abnormal conduction zone and contact area/low voltage area}

All patients underwent ECG-gated 64-slice multidetector CT (SOMATOM Definition Edge; Siemens) with slice acquisition thickness set at $0.5 \mathrm{~mm}$. The CoAs (ascending aorta-anterior LA, descending aorta-posterior LA, and vertebrae-posterior LA) were assessed using CT. The anatomical distance between those structures and the LA was calculated. CoA was defined as the distance between any external structures and LA was within $3 \mathrm{~mm} .{ }^{13}$ Subsequently, we traced the CoAs inside the LA on each 2-mm CT slice, and three particular areas were created on the 3-D mapping system (Figure 2 ). The CoA size was manually measured on each segment. The anatomical relationship between the ACZ and CoAs/LVA at each cut-off value was analyzed (Figure 2 ). The overlapping rate of the ACZ and CoAs/LVA was assessed for each patient (Figure 1, 2 ).

\section{Statistical analysis}

Continuous variables (e.g., age) were given as mean $\pm \mathrm{SD}$, and were analyzed with $\mathrm{t}$ tests if they conformed to a normal distribution and Mann-Whitney $U$ tests if not. Comparisons between more than 2 groups made with one-way analysis of variance. Categorical variables (e.g., gender) were compared using the $\chi^{2}$ test or the Fisher exact test. $\mathrm{P}<0.05$ was considered significant. All analyses were performed using JMP Pro statistical software (version 10, SAS Inc, Cary, NC) and Microsoft Excel.

\section{Results}

\section{Patients ${ }^{6}$ characteristics}

All 100 patients were enrolled in the study according to specific inclusion and exclusion criteria. The average number of recorded points was $4580 \pm 1035$ (interquartile range; IQR 2466-7711). Of the 100 patients, the ACZ was observed in 95 patients (95\%), and the ACZ was linearly distributed (Figure 1 ). To investigate the characteristics of patients with ACZ, we divided all patients into two groups as follows: non-ACZ group $(\mathrm{n}=5)$ and ACZ group $(\mathrm{n}=95)$. Patients' characteristics are listed in Table $\mathbf{1}$. All five patients in the non-ACZ group had PAF. Patients in the ACZ group had significantly elevated body mass index (BMI), higher prevalence of hypertension, and larger LA diameter/BSA than those in the non-ACZ group. Baseline medication therapy and the other TTE findings did not differ between the non-ACZ group and the ACZ group.

\section{Type of atrial fibrillation and abnormal conduction zone}

Table 2 lists the procedural findings of the study group. We divided all patients into two groups as follows: PAF group ( $\mathrm{n}=51)$ and non-PAF group $(\mathrm{n}=49)$. Patients in the non-PAF group had a wider total LA surface area, larger number of mapping points, longer global LA activation time, lower global LA voltage, higher incidence of ACZ, longer ACZ length, higher incidence of LVA and higher burden of LVA than those in the PAF group.

\section{Left atrial size and abnormal conduction zone}

For further analysis, all patients were divided into three groups as follows: normal / mildly enlarged LA group $(n=68)$, moderately enlarged LA group $(n=21)$, and severely enlarged LA group $(n=11)$ according to LA diameter/BSA. Larger LA size was associated with lower global LA voltage (normal/mildly enlarged LA group: $5.8 \pm 2.2 \mathrm{mV}$, moderately enlarged LA group: $5.0 \pm 2.1 \mathrm{mV}$, severely enlarged LA group: $3.2 \pm 1.5 \mathrm{mV}$, $\mathrm{P}=0.0011$ ). The incidence and number of $\mathrm{ACZ}$ did not differ among the three groups. A longer ACZ was significantly associated with a larger LA size (Table 2 , normal/mildly enlarged LA group: $49.4 \pm 40.2 \mathrm{~mm}$, moderately enlarged LA group: $62.7 \pm 45.6 \mathrm{~mm}$, severely enlarged LA group: $77.8 \pm 38.3 \mathrm{~mm}, \mathrm{P}=0.0469$ ).

\section{Incidence and distribution of abnormal conduction zone}

Figure 3 shows the incidence and distribution of the ACZ in each LA region. Of the 100 patients, the ACZ was most frequently observed in the anterior wall region $(77 \%)$. Moreover, a tendency of increased 
$\mathrm{ACZ}$ in the anterior wall region was shown in the other categories (PAF, non-PAF, normal/mildly enlarged LA, moderately enlarged LA, and severely enlarged LA). Compared to the PAF group, the incidence of the ACZ was higher in the non-PAF group in global LA ( $100 \%$ vs $90 \%, \mathrm{P}=0.0245)$, anterior ( $86 \%$ vs $69 \%$, $\mathrm{P}=0.0424)$, posterior ( $20 \%$ vs $4 \%, \mathrm{P}=0.0112)$, and roof $(59 \%$ vs $31 \%, \mathrm{P}=0.0052)$. A larger LA size was significantly associated with a higher incidence of a ACZ in anterior, posterior, inferior, and roof (Figure 3

). Figure 4shows the incidence of the ACZ and LVA in each group. In the 100 patients, the ACZ (95\%) had a significantly higher incidence rate than for each LVA (mild LVA: 78\%, moderate LVA: $56 \%$, severe LVA: 20\%). Furthermore, these tendencies were also observed in the other groups (Figure 4 ).

\section{Anatomical relationship between abnormal conduction zone and contact area/low voltage area}

Table 2 lists the anatomical relationship between the ACZ and CoAs/LVA. The CoAs were found in all patients. A total of $185 \mathrm{CoAs}$ (average CoA per patient: $6.0 \pm 4.1 \mathrm{~cm}^{2}$ ) were identified. A CoA was found in each of the three representative regions, ascending aorta-anterior LA $\left(3.2 \pm 1.8 \mathrm{~cm}^{2}, 100\right.$ of 100 patients, $100 \%)$, descending aorta-posterior LA $\left(0.6 \pm 1.3 \mathrm{~cm}^{2}, 26\right.$ of 100 patients, $\left.26 \%\right)$, and vertebrae-posterior LA $\left(2.1 \pm 2.7 \mathrm{~cm}^{2}, 58\right.$ of 100 patients, $\left.58 \%\right)$. CoA and number of CoA did not differ between the PAF group and the non-PAF group. The overlapping rate of LVA and ACZ showed a tendency to increase in the nonPAF group compared with the PAF group. (Table 2 , mild LVA: $60.6 \pm 34.0 \%$ vs $42.1 \pm 36.2 \%, \mathrm{P}=0.0099$; moderate LVA: $41.4 \pm 33.3 \%$ vs $24.8 \pm 30.4 \%, \mathrm{P}=0.0109$, severe LVA: $18.9 \pm 23.5 \%$ vs $10.3 \pm 19.9 \%, \mathrm{P}=0.0502)$. On the other hand, the overlapping rate of CoAs and ACZ did not differ between the PAF $(26.5 \pm 33.1 \%)$ and non-PAF $(24.6 \pm 21.6 \%)$ groups. A larger LA size was associated with a higher overlapping rate of LVA (at each cut-off value) and ACZ. In contrast, the overlapping rate of CoAs and ACZ did not differ among the normal/mildly, moderately, and severely enlarged LA group.

\section{Discussion}

This was a prospective observational study of the characteristics of the ACZ in patients with AF using the databases from two centers. The main findings were:

(1) the ACZ was observed in $95 \%$ patients, and all ACZ was linearly distributed,

(2) the ACZ was most frequently observed in the anterior wall region $(77 \%)$,

(3) a longer ACZ was significantly associated with a larger LA size and a prevalence of non-PAF,

(4) a larger LA size was associated with a higher overlapping rate of LVA and ACZ, and

(5) a larger LA size was not associated with a higher overlapping rate of CoAs and ACZ.

With the development of the 3-D mapping system, there has been significant focus on the conduction pattern of the LA. Recently, Yamaguchi et al reported that a difference in bipolar voltage mapping between ring catheter and HD Grid. ${ }^{14}$ They stated that the HD Grid can create a voltage map that is independent of propagation direction and can assess more precise LA conduction pattern. To date, several studies have reported on the conduction abnormalities of the LA. However, the conduction abnormalities varied between patients, and there were various methods of mapping and different definitions of the ACZ in each study.

A limited number of studies have reported on conduction abnormalities at the LA in humans. Markides et al studied the LA activation during SR in 19 AF patients with noncontact mapping system. They found that line of conduction block running vertically in the LA posterior wall was observed in all patients. Moreover, they reported that the anterior line extending from the anterior mitral valve annuls toward the left atrial appendage was observed in 9 patients $(47 \%) .{ }^{15}$ Mouws et al investigated the conduction abnormalities during sinus rhythm only at the LA posterior wall using epicardial mapping in 268 patients who underwent cardiac surgery. ${ }^{6}$ They showed that conduction abnormalities occurred in $90 \%$ of patients. Moreover, in their study, AF episode was associated with a higher incidence of conduction abnormality, larger number of lines, and longer lines. Roberts-Thomson et al reported the conduction properties of the posterior LA during SR using epicardial mapping in 34 patients who underwent cardiac surgery. ${ }^{16}$ In their study, 5 patients (15\%) had an ACZ running vertically down the posterior LA. The results of our study showed that the ACZ was observed 
in 95\% patients, and all ACZ was linearly distributed, similar to the results of previous studies. On the other hand, the prevalence of the ACZ was higher in our study than those in previous studies although our study did not include patients with structural heart disease. This discrepancy in findings might be due to different patient inclusion criteria, methods of mapping (epicardial or endocardial mapping), mapping catheter, and definition of the ACZ. However, very few studies have focused on the ACZ using the HD Grid. Further investigation on the ACZ is warranted.

In our study, the ACZ was associated with a non-PAF, BMI, hypertension, and LA diameter/BSA. These factors are well known predictors which increase the prevalence of AF. It has also been established that non-PAF increases predisposition to LA remodeling in a so-called "AF begets AF" manner. A recent study showed that patients with AF had a higher incidence of conduction abnormality, larger number of lines, and longer lines than those without AF. ${ }^{6}$ These findings support our results indicating that existence of ACZ is associated with non-PAF, BMI, hypertension, and LA diameter/BSA. Moreover, we found that a longer ACZ was significantly associated with a larger LA size. The association between LA size and ACZ yielded mixed results in previous studies. Roberts-Thomson et al reported that the line of conduction delay was most marked in conditions associated with greater LA enlargement. ${ }^{16}$ In contrast to our results, the other study showed that LA dilation was not predictor of long line of conduction abnormalities. ${ }^{6}$ Furthermore, their patients had a larger LA size compared with our patients because they included structural heart disease such as valvular heart disease. This discrepancy in findings may be explained by the different baseline LA size.

It is well-known that the ACZ plays an important role in atrial tachyarrhythmias, however, the causes of the ACZ are still unclear. We considered the following mechanisms of the ACZ. First, the ACZ might be the result of an abrupt change in myocardial fiber direction. A previous study showed that all hearts have subendocardial fibers, and the longitudinal fibers were arranged in broad bands. Moreover, they found that the most obvious broad band of longitudinal fibers was formed by the "septopulmonary bundle". ${ }^{17}$ Markides et al also reported that abnormal conduction was associated with a change in these myocardial fiber direction. ${ }^{15}$ In fact, the location of the ACZ shown in Figure 1 matches the septopulmonary bundle at the anterior wall. On the other hand, the incidence of the ACZ in the posterior wall in our study was lower than in the previous study. This discrepancy in the incidence of the ACZ may be explained by the different activation flow. In our study, the activation in the posterior wall was activated vertically from the roof and bottom because mapping was performed during high right atrial pacing (Supplementary video ). Therefore, the incidence of the ACZ in the posterior wall was low in our study, and the pacing from different site might have led to the different distribution of the ACZ. Second, the compression from the external organs might induce the ACZ. Mayyas et al found that endothelin-1, which promote myocyte hypertrophy and interstitial fibrosis, is an important factor in AF development and persistence. ${ }^{18}$ They also mentioned that greater wall stress may produce higher levels of endothelin- 1 because this factor is stretch mediated. Based on this report, we hypothesized that externally-mediated wall stress leads to regional differences in the ACZ. In our study, the overlapping rate of LVA (mild and moderate) and ACZ was higher than that of CoAs and ACZ. Therefore, the ACZ is associated with an LVA rather than a CoAs. These findings indirectly suggest that the ACZ might be a precursor to LVA.

\section{Clinical implication}

To the best of our knowledge, this is the first study to identify a relationship between the ACZ and the LVA/LA anatomical CoAs in patients with AF. To improve the outcome after CA for AF, a more detailed understanding of the LA activation is essential. Consequently, the assessment of the ACZ might have a critical implication in risk stratification of atrial tachyarrhythmias recurrence after the CA, and could be helpful in providing an appropriate new strategy for CA. A prior study suggested that the LA posterior wall plays an important role in both initiation and the maintenance of AF, and a recent systematic review showed the efficacy of posterior wall isolation for AF. ${ }^{19}$ These findings indirectly suggest that the posterior ACZ may play an important role in patients with AF. However, we did not investigate the association between the ACZ and the recurrence of AF. Further study will be necessary to determine which position of the ACZ is important in $\mathrm{AF}$. 


\section{Limitation}

There were several limitations to our study. First, this is a study with a small number of patients. Second, it is possible that the LVA and ACZ varied with heart rate or pacing site. Atrial tachyarrhythmias occur by premature atrial beat during sinus rhythm where electrical activity originating from the sinus node spreads towards the superior vena cava and right atrial appendage. Moreover, a previous study reported that LA activation pattern did not differ regardless of site of earliest LA breakthrough. ${ }^{15}$ Hence, using LA activation during high right atrial pacing is acceptable. Third, our study completed 3-D mapping after the PV isolation. Fourth, there was no control group in our study. To evaluate our hypothesis, a control group is crucially important. Lastly, the criteria for defining the ACZ was different from previous studies.

\section{Conclusion}

The abnormal conduction zone reflects LA electrical remodeling and may be a precursor finding of the low voltage area and not LA contact areas in patients with atrial fibrillation.

Conflict of interest: none declared

\section{References}

1. Atul Verma, Chen-yang Jiang, Timothy R Betts et al. Approaches to Catheter Ablation for Persistent Atrial Fibrillation. N Engl J Med 2015;372:1812-1822.

2. Takanori Yamaguchi, Takeshi Tsuchiya, Shiro Nakahara et al. Efficacy of Left Atrial Voltage-Based Catheter Ablation of Persistent Atrial Fibrillation. J Cardiovasc Electrophysiol 2016;27:1055-1063.

3. Peter M Kistler, Prashanthan Sanders, Miodrag Dodic et al. Atrial Electrical and Structural Abnormalities in an Ovine Model of Chronic Blood Pressure Elevation After Prenatal Corticosteroid Exposure: Implications for Development of Atrial Fibrillation. Eur Heart J 2006;27:3045-3056.

4. Andrew W Teh, Peter M Kistler, Geoffrey Lee et al. Long-term Effects of Catheter Ablation for Lone Atrial Fibrillation: Progressive Atrial Electroanatomic Substrate Remodeling Despite Successful Ablation. Heart Rhythm 2012;9:473-480.

5. R Ferrari, M Bertini, C Blomstrom-Lundqvist et al. An Update on Atrial Fibrillation in 2014:From Pathophysiology to Treatment. Int J Cardiol 2016;203:22-29.

6. Elisabeth M J P Mouws, Lisette J M E van der Does, Charles Kik et al. Impact of the Arrhythmogenic Potential of Long Lines of Conduction Slowing at the Pulmonary Vein Area. Heart Rhythm 2019;16:511519.

7. Yuichi Hori, Shiro Nakahara, Tohru Kamijima et al. Influence of Left Atrium Anatomical Contact Area in Persistent Atrial Fibrillation-Relationship Between Low-Voltage Area and Fractionated Electrogram. Circ J 2014;78:1851-1857.

8. Roberto M Lang, Michelle Bierig, Richard B Devereux et al. Recommendations for Chamber Quantification: A Report from the American Society of Echocardiography's Guidelines and Standards Committee and the Chamber Quantification Writing Group, Developed in Conjunction with the European Association of Echocardiography, a Branch of the European Society of Cardiology. J Am Soc Echocardiogr 2005;18:1440-1463.

9. Yazhou Lin, Bing Yang, Fermin C Garcia et al. Comparison of Left Atrial Electrophysiologic Abnormalities During Sinus Rhythm in Patients with Different Type of Atrial Fibrillation. J Interv Card Electrophysiol 2014;39:57-67.

10. Xin-Hua Wang, Zheng Li, Jia-Liang Mao, Min-Hua Zang, Jun Pu. Low Voltage Areas in Paroxysmal Atrial Fibrillation: The Prevalence, Risk Factors and Impact on the Effectiveness of Catheter Ablation. Int J Cardiol 2018;269:139-144.

11. Atul Verma, Oussama M Wazni, Nassir F Marrouche et al. Pre-existent Left Atrial Scarring in Patients Undergoing Pulmonary Vein Antrum Isolation: An Independent Predictor of Procedural Failure. J Am Coll Cardiol 2005;45:285-292.

12. Hans-Ruprecht Neuberger, Ulrich Schotten, Yuri Blaauw et al. Chronic Atrial Dilation, Electrical Remodeling, and Atrial Fibrillation in the Goat. J Am Coll Cardiol 2006;47:644-653. 
13. Shiro Nakahara, Takanori Yamaguchi, Yuichi Hori et al. Spatial Relation Between Left Atrial Anatomical Contact Areas and Circular Activation in Persistent Atrial Fibrillation. J Cardiovasc Electrophysiol 2016;27:515-523.

14. Takanori Yamaguchi, Akira Fukui, Koichi Node. Bipolar Voltage Mapping for the Evaluation of Atrial Substrate: Can We Overcome the Challenge of Directionality? J Atr Fibrillation 2019;11:2116.

15. Vias Markides, Richard J Schilling, Siew Yen Ho, Anthony W C Chow, D Wyn Davies, Nicholas S Peters. Characterization of Left Atrial Activation in the Intact Human Heart. Circulation 2003;107:733739.

16. Kurt C Roberts-Thomson, Irene H Stevenson, Peter M Kistler et al. Anatomically Determined Functional Conduction Delay in the Posterior Left Atrium Relationship to Structural Heart Disease. J Am Coll Cardiol 2008;51:856-862.

17. Papez J. Heart musculature of the atria. Am J Anat. 1920;27:255-285.

18. Fadia Mayyas, Mark Niebauer, Andrew Zurick et al. Association of Left Atrial endothelin-1 with Atrial Rhythm, Size, and Fibrosis in Patients with Structural Heart Disease. Circ Arrhythm Electrophysiol 2010;3:369-379.

19. Anand Thiyagarajah, Kadhim Kadhim, Dennis H Lau et al. Feasibility, Safety, and Efficacy of Posterior Wall Isolation During Atrial Fibrillation Ablation:A Systematic Review and Meta-Analysis. Circ Arrhythm Electrophysiol 2019;12:e07005.
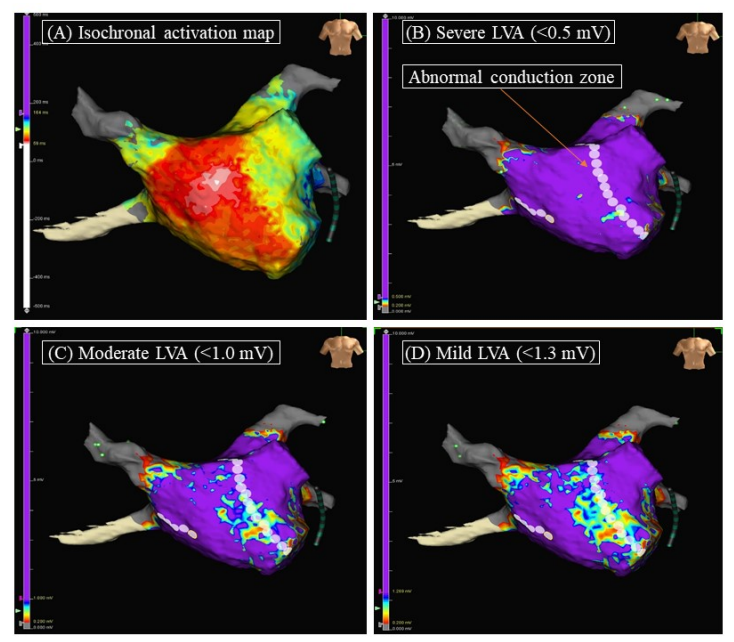

Figure 1. (A) Isochronal map in the left atrium during high right atrial pacing. (B), (C), (D) Comparison of voltage map in the left atrium during high right atrial pacing in a different voltage cut-off value of $<0.5$ $\mathrm{mV},<1.0 \mathrm{mV}$, and $<1.3 \mathrm{mV}$. LVA = low voltage area. 

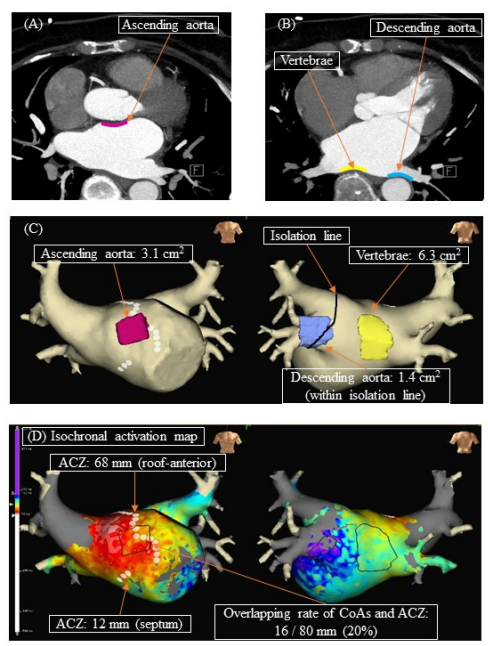

Figure 2. (A), (B), (C) Measurement of left atrial CoAs from the external structures. (D) Representative case of measurement of overlapping rate of $\mathrm{CoAs}$ and $\mathrm{ACZ}$. ACZ=abnormal conduction zone; $\mathrm{CoAs}=$ contact areas.

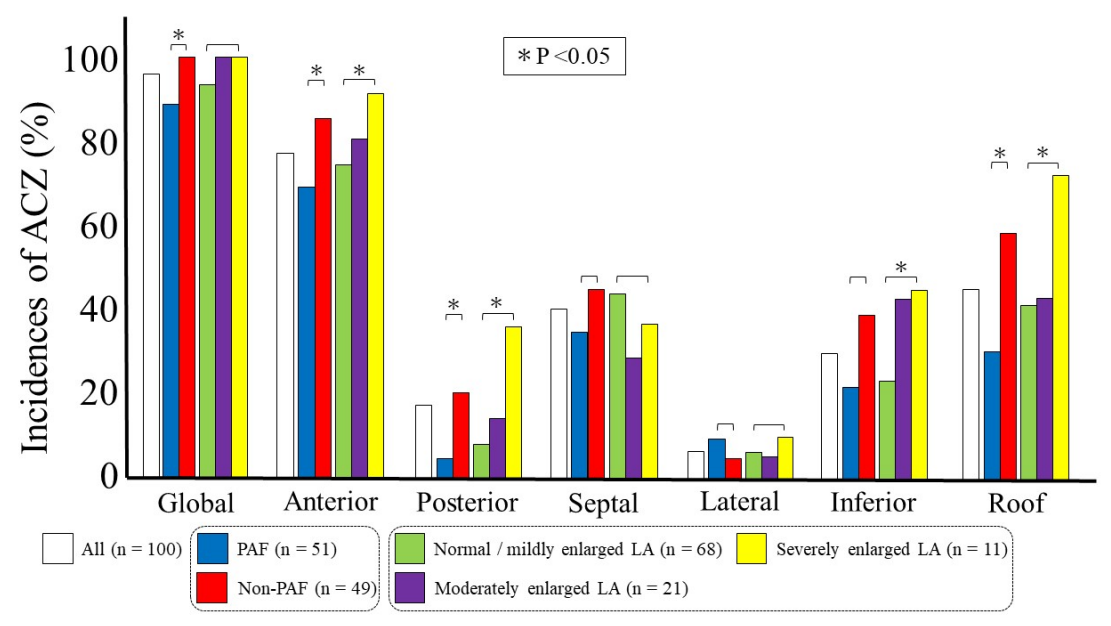

Figure 3. Incidence and distribution of the $\mathrm{ACZ}$ in each LA region. $\mathrm{ACZ}=$ abnormal conduction zone; $\mathrm{LA}=$ left atrium; $\mathrm{PAF}=$ paroxysmal atrial fibrillation. 


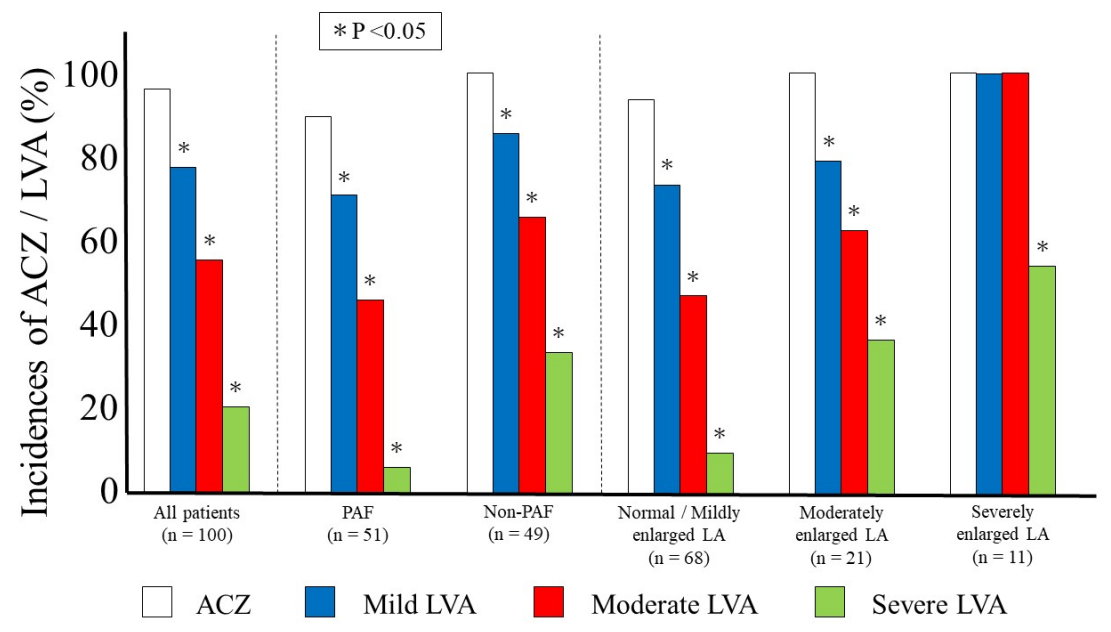

Figure 4. Incidence of the ACZ and LVA in each cut-off value. ACZ=abnormal conduction zone; LA=left atrium; LVA=low voltage area.

${ }^{*} \mathrm{P}<0.05$ for the comparison of the ACZ vs. mild/moderate/severe LVA.

Male, n (\%)

Age (year)

Body mass index $\left(\mathrm{kg} / \mathrm{m}^{2}\right)$

Body surface area $\left(\mathrm{m}^{2}\right)$

Non-paroxysmal AF, n (\%)

Hypertension, n (\%)

Diabetes mellitus, n (\%)

Prior stroke/TIA, n (\%)

$\mathrm{CHADS}_{2}$ score

Brain natriuretic peptide $(\mathrm{pg} / \mathrm{mL})$

Creatinine $(\mathrm{mg} / \mathrm{dL})$

Medication therapy

Beta blocker, n (\%)

Angiotensin converting enzyme inhibitor, n (\%)

Angiotensin receptor blocker, n (\%)

Calcium blocker, n (\%)

Statin, n (\%)

Antiplatelet, n (\%)

Direct oral anticoagulant, n (\%)

Antiarrhythmic drug, n (\%)

Echocardiographic findings

LA diameter ( $\mathrm{mm}$ )

LA diameter/Body surface area $\left(\mathrm{cm} / \mathrm{m}^{2}\right)$

$\mathrm{E}$ wave $(\mathrm{cm} / \mathrm{s})$

Deceleration time $(\mathrm{ms})$

LV diameter during end diastole ( $\mathrm{mm}$ )

LV hypertrophy, n (\%)

LV ejection fraction (\%)

$\mathrm{E} / \mathrm{e}^{6}$ ratio

\begin{tabular}{lll} 
All patients $(\mathrm{n}=100)$ & 1. Non-ACZ group $(\mathrm{n}=5)$ & 2. ACZ group $(\mathrm{n}=95)$ \\
$66(66 \%)$ & $3(60 \%)$ & $63(66 \%)$ \\
$67.9 \pm 9.9$ & $63.6 \pm 11.4$ & $68.1 \pm 9.8$ \\
$25.2 \pm 3.9$ & $21.1 \pm 2.0$ & $25.4 \pm 3.9$ \\
$1.7 \pm 0.2$ & $1.6 \pm 0.2$ & $1.8 \pm 0.2$ \\
$49(49 \%)$ & $0(0 \%)$ & $49(52 \%)$ \\
$64(64 \%)$ & $1(20 \%)$ & $63(66 \%)$ \\
$19(19 \%)$ & $0(0 \%)$ & $19(20 \%)$ \\
$12(12 \%)$ & $1(20 \%)$ & $11(12 \%)$ \\
$1.6 \pm 1.2$ & $0.8 \pm 1.8$ & $1.6 \pm 1.2$ \\
$120 \pm 146$ & $50 \pm 51$ & $125 \pm 148$ \\
$0.98 \pm 0.86$ & $0.99 \pm 0.46$ & $0.98 \pm 0.88$ \\
& & \\
$50(50 \%)$ & $1(20 \%)$ & $49(52 \%)$ \\
$5(5 \%)$ & $0(0 \%)$ & $5(5 \%)$ \\
$39(39 \%)$ & $1(20 \%)$ & $38(40 \%)$ \\
$39(39 \%)$ & $1(20 \%)$ & $38(40 \%)$ \\
$37(37 \%)$ & $0(0 \%)$ & $37(39 \%)$ \\
$13(13 \%)$ & $0(0 \%)$ & $13(14 \%)$ \\
$94(94 \%)$ & $4(80 \%)$ & $90(95 \%)$ \\
$35(35 \%)$ & $2(40 \%)$ & $33(35 \%)$ \\
& & \\
$43 \pm 7$ & $34 \pm 5$ & $44 \pm 6$ \\
$2.5 \pm 0.4$ & $2.1 \pm 0.3$ & $2.5 \pm 0.4$ \\
$77 \pm 22$ & $67 \pm 16$ & $78 \pm 22$ \\
$182 \pm 53$ & $199 \pm 29$ & $181 \pm 54$ \\
$49 \pm 5$ & $46 \pm 4$ & $49 \pm 5$ \\
$14(14 \%)$ & $0(0 \%)$ & $14(15 \%)$ \\
$64 \pm 9$ & $67 \pm 4$ & $63 \pm 9$ \\
$10.7 \pm 3.9$ & $7.7 \pm 0.8$ & $10.9 \pm 4.0$ \\
\hline & &
\end{tabular}


Table 1. Baseline characteristics

Values are given as no. (\%) or mean \pm SD.

$\mathrm{ACZ}=$ abnormal conduction zone; $\mathrm{LA}=$ left atrial; $\mathrm{LV}=$ left ventricular; TIA=transient ischemic attack.

Total surface area of LA $\left(\mathrm{cm}^{2}\right)$

Number of mapping point

Global LA activation time (ms)

Global LA voltage $(\mathrm{mV})$

Incidence of $\mathrm{ACZ}, \mathrm{n}(\%)$

Length of $\mathrm{ACZ}(\mathrm{cm})$

Number of ACZ

$\mathrm{CoA}\left(\mathrm{cm}^{2}\right)$

Number of CoA

Overlapping rate of CoAs and ACZ (\%)

Incidence of mild LVA $(<1.3 \mathrm{mV}), \mathrm{n}(\%)$

Burden of mild LVA $\left(\mathrm{cm}^{2}\right)$

Overlapping rate of mild LVA and ACZ (\%)

Incidence of moderate LVA $(<1.0 \mathrm{mV}), \mathrm{n}(\%)$

Burden of moderate LVA $\left(\mathrm{cm}^{2}\right)$

Overlapping rate of moderate LVA and ACZ (\%)

Incidence of severe LVA $(<0.5 \mathrm{mV})$, n $(\%)$

Burden of severe LVA $\left(\mathrm{cm}^{2}\right)$

Overlapping rate of severe LVA and ACZ (\%)

$\begin{array}{ll}\text { All patients }(\mathrm{n}=100) & 1 . \text { PAF group }(\mathrm{n}=51) \\ 78.8 \pm 19.7 & 69.2 \pm 13.6 \\ 4580 \pm 1035 & 4331 \pm 939 \\ 112 \pm 21 & 101 \pm 15 \\ 5.3 \pm 2.2 & 6.2 \pm 2.0 \\ 95(95 \%) & 46(90 \%) \\ 5.48 \pm 4.00 & 3.81 \pm 2.95 \\ 2.2 \pm 1.4 & 1.6 \pm 1.0 \\ 6.0 \pm 4.1 & 5.5 \pm 4.2 \\ 1.9 \pm 0.6 & 1.8 \pm 0.5 \\ 25.6 \pm 28.0 & 26.5 \pm 33.1 \\ 78(78 \%) & 36(71 \%) \\ 19.1 \pm 16.5 & 13.5 \pm 13.3 \\ 51.2 \pm 36.2 & 42.1 \pm 36.2 \\ 56(56 \%) & 23(45 \%) \\ 10.0 \pm 10.7 & 6.4 \pm 7.8 \\ 32.9 \pm 32.8 & 24.8 \pm 30.4 \\ 20(20 \%) & 3(6 \%) \\ 2.9 \pm 5.3 & 1.0 \pm 1.9 \\ 14.6 \pm 22.0 & 10.3 \pm 19.9\end{array}$

2. Non-PAF group $(\mathrm{n}=4$ $88.8 \pm 20.2$ $4840 \pm 1076$

$124 \pm 21$

$4.4 \pm 2.0$

$49(100 \%)$

$7.22 \pm 4.23$

$2.6 \pm 1.1$

$6.5 \pm 3.9$

$1.9 \pm 0.7$

$24.6 \pm 21.6$

$42(86 \%)$

$24.9 \pm 17.7$

$60.6 \pm 34.0$

$33(67 \%)$

$13.8 \pm 12.1$

$41.4 \pm 33.3$

$17(35 \%)$

$5.0 \pm 6.8$

$18.9 \pm 23.5$

Table 2. Procedural characteristics

Values are given as no. (\%) or mean \pm SD.

$\mathrm{ACZ}=$ abnormal conduction zone; $\mathrm{CoA}=$ contact area; $\mathrm{LA}=$ left atrium; $\mathrm{LVA}=\mathrm{low}$ voltage area; $\mathrm{PAF}=$ paroxysmal atrial fibrillation.
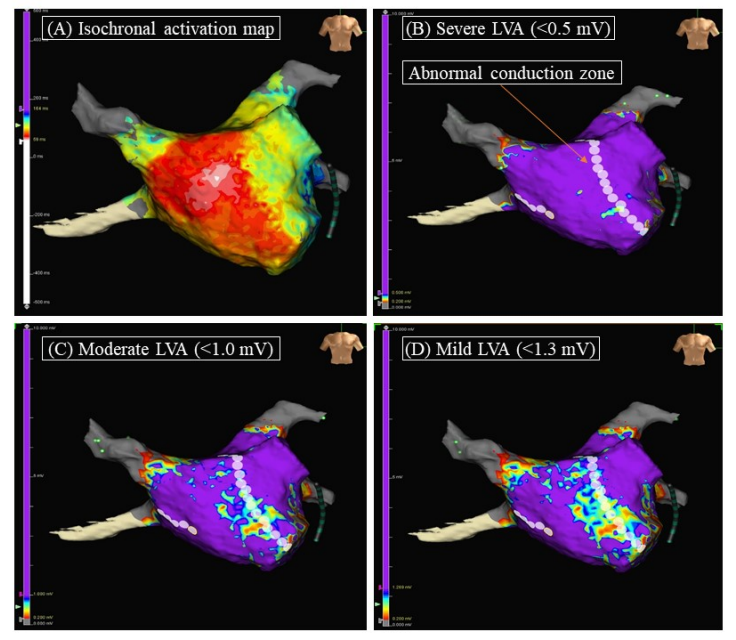

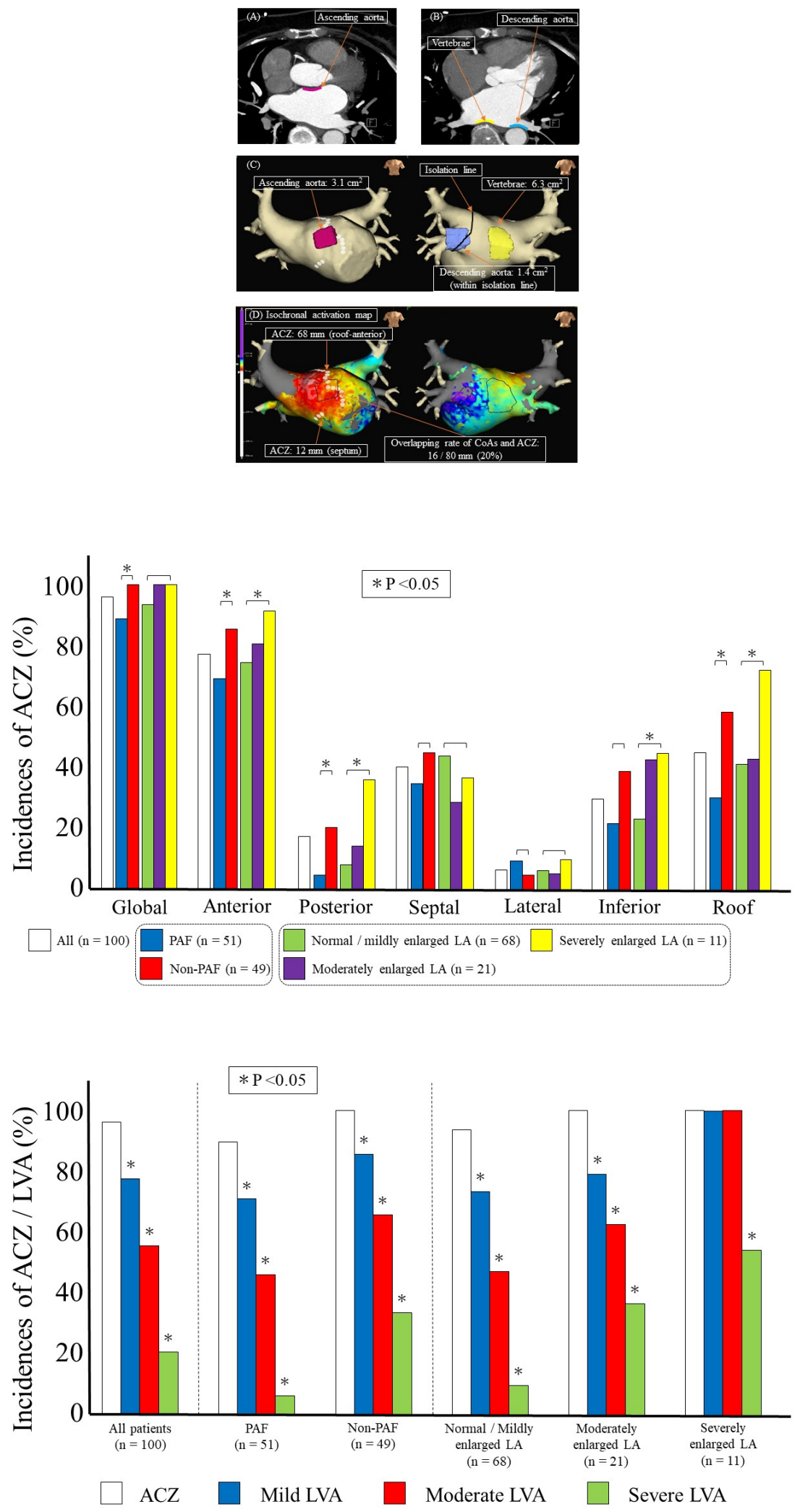


\section{Hosted file}

Table1.docx available at https://authorea.com/users/347301/articles/473046-is-the-abnormalconduction-zone-of-the-left-atrium-a-precursor-to-a-low-voltage-area-in-patients-withatrial-fibrillation

\section{Hosted file}

Table2.docx available at https://authorea.com/users/347301/articles/473046-is-the-abnormalconduction-zone-of-the-left-atrium-a-precursor-to-a-low-voltage-area-in-patients-withatrial-fibrillation 